\title{
High Gain Compact Strip and Slot UWB Sinuous Antennas
}

\author{
Elson Agastra, Leonardo Lucci, Giuseppe Pelosi, and Stefano Selleri \\ Department of Electronics and Telecommunications, University of Florence, 3 Via Santa Marta, 50139 Florence, Italy
}

Correspondence should be addressed to Stefano Selleri, stefano.selleri@unifi.it

Received 5 October 2012; Accepted 17 October 2012

Academic Editor: Renato Cicchetti

Copyright (C) 2012 Elson Agastra et al. This is an open access article distributed under the Creative Commons Attribution License, which permits unrestricted use, distribution, and reproduction in any medium, provided the original work is properly cited.

Three ground-backed compact strip-and slot-sinuous antennas are analyzed. Proposed configuration allows for a single lobe, polarization-versatile, high efficiency, and ultrawide band antenna not needing a cumbersome lossy back cavity typical of conventional single-lobe sinuous antennas. Simulations show attained performances as well as tuning possibilities.

\section{Introduction}

In the last two decades, the advances in communication technology, in particular mobile communications, have drastically changed the lifestyles of people. Antenna engineers have contributed to this wireless revolution by designing high performance antennas for fixed base-stations and mobile portable terminals. The demands placed on mobile communication systems have increased remarkably, so antenna designers face many challenges: small or tiny sizes, low- or ultra-low-profiles, wide- or ultra-wide-operating bandwidths, multiple operative modes, polarization agility, and, of course, simple fabrication and low cost. As a result, antenna research and development have become a hot topic in both academia and industry.

Indeed ultra-wideband antennas are needed in many engineering applications not only in telecommunications, for example, in radar and direction-finding systems, through wall imaging, anti-collision radars, radio astronomy, and biomedical systems.

Hot topics in ultra-wideband antenna are printed structures [1] as the small size and light weight are mandatory to the antenna success. Many researches are concentrated on increasing the band width of the printed antenna $[2,3]$. In any case, even if large bands are obtained, polarization purity and agility are not investigated [4-10]. Many of these researches have in common the development of a slotrather than patch-structure.

Increasing the requirements for bandwidth, uniform antenna gain, and polarization agility, the antenna engineers need to consider the principles at the basis of frequency independent structures. Frequency-independent antennas exhibit some self-scaling property in their geometry where polarization, radiation pattern, and input impedance remain unchanged or vary with a limited, predictable, periodicity, over large bandwidths [11]. Spiral antennas are classified into different types: Archimedean spiral, logarithmic spiral, square spiral, star spiral, and so forth. Archimedean and logarithmic spirals are very popular configurations and among the most analyzed frequency independent antennas. Many research is concentrated on reducing spiral antenna size [12], but as the spiral antenna radiates two main lobes, one above and one below its plane, when a single radiation lobe is needed, an absorbing cavity is used to prevent radiation in one of the two half spaces [11].

To the spiral antenna, the sinuous antenna is a good alternative. It shares many of its features: it is planar, broadband and presents two lobes. On the other hand, while spirals have a circular polarization, sinuous antennas exhibit a linear polarization in their two-arm version, and dual linear polarization in their four arms version $[11,13]$. Indeed the two available orthogonal linear polarizations can be used for polarization diversity in transmit/receive as well as to produce simultaneously a Left Hand Circular Polarization (LHCP) and a Right Hand Circular Polarization (RHCP) [11-15]. The antenna lower and higher frequency operation limits are determined, respectively, by the outer and inner diameters of the structure.

As the theoretical sinuous antenna radiates both above and below its plane, a broadband absorbing cavity is typically used to eliminate the radiation in one direction as any reflections from a metallic bottom would couple back 
to the antenna feed and radiate with the opposite sense of polarization, spoiling polarization purity. Typically, the absorber-filled cavity for this antenna has a depth on the order of the antenna radius. Although the lossy cavity drastically reduces antenna efficiency below $50 \%$ and makes the complete structure quite bulky, most commercial sinuous antenna are realized this way $[16,17]$.

Better results might be reached via a stepped or conical cavity, so as to keep the back wall at a quarter wavelength from the active region. This can be done more compact by properly loading with a high permittivity material the cavity or by exploiting metamaterials; some interesting results for this two configurations are presented in the following. If, on the other hand, a flat ground is preferred, then a stepped permittivity can be used in the substrate to vary the electrical distance of the planar ground. Both these possibilities will be addressed in the following. In any case these configuration still needs a backing, possibly lossy, cavity of nonnegligible thickness.

A sinuous antenna in slot configuration is not burdened with most of these issue. Additionally, while the slot spiral also radiates bidirectionally, a deep, absorbing cavity is not necessary to force unidirectional radiation. In fact, a very shallow reflecting cavity is sufficient and leads to an appreciable increase in gain. The resulting complete antenna is very thin, making mounting much easier. Notwithstanding its better behavior, little research has been carried out for slot-sinuous antennas [18-20].

This paper is focused on the design of three four-arm sinuous antennas, enabling polarization agility if backed by an appropriate feeding network, and with increased efficiency (with respect to the lossy cavity backed one). Two of the proposed configurations exhibit a strip sinuous over a properly design backing cavity, while the last is a slotsinuous configuration with a closer ground. In this latter configuration, by varying the distance from the ground, some control over the half power beam width (HPBW) can be obtained.

\section{Sinuous Antenna Description}

The standard sinuous antenna is composed of at least two arms, where each is composed by cells. The description of printed sinuous antenna can be founded in the DuHamel patent [13].

The slot-sinuous antenna is essentially the complementary structure of a conventional sinuous antenna. The fundamental analytical expression that permits to realize the sinuous antenna geometry is reported in (1)

$$
\varphi=(-1)^{p} \alpha_{p} \sin \left[\frac{180 \ln \left(r / R_{p}\right)}{\ln \left(\tau_{p}\right)}\right],
$$

where $r$ and $\varphi$ are polar coordinates of the points on the curve relative to the $p$ th cell, whose domain is $R_{p+1} \leq r \leq R_{p}$. Cell1 corresponds to the outermost cell, with radius $R_{1}$, and, as a recursive rule, $R_{p}=\tau_{p-1} R_{p-1} . \alpha_{p}$ and $\tau_{p}$ are appropriate constants, describing the angular width and the scale factor of the antenna, much alike a printed log-periodic antenna.
The curve obtained by (1) is then rotated by $\pm \delta$ around the $z$ axis to define the boundaries of one of the printed arms (Figure 1). As we are interested in the four arm structure, this arm is finally duplicated and rotated of $90^{\circ}, 180^{\circ}$, and $270^{\circ}$ about the origin creating the complete four arms antenna. For the slot antenna, the complementary structure is considered, where the above described surfaces are etched on a ground plane, rather than metalized over a substrate.

To validate the chosen layout, a FEM analysis of the proposed structure is performed via Ansoft HFSS, both in free space and with a backing cavity. The geometry parameters considered here for all configurations are: $R_{1}=$ $14 \mathrm{~mm}, \alpha_{p}=\alpha=45^{\circ}, \tau_{p}=\tau=0.77, \delta_{p}=\delta=22.5^{\circ}$, $P=11$ (total number of cells). The $\alpha$ and $\delta$ chosen lead to a self-complementary structure. The chosen values for $R_{1}$ and $P$ lead to a theoretical band spanning from $6 \mathrm{GHz}$ to $18 \mathrm{GHz}$ ( $3: 1$ frequency band). It is worth noticing that the slot configuration needs an outer ground ring, so only for this case, while the radius of the antenna is still $14 \mathrm{~mm}$ the radius of the whole structure extends to $18 \mathrm{~mm}$.

Both the strip-and slot-sinuous antennas inherently radiates bidirectionally.

\section{Strip Sinuous with Compact Cavity}

Classical sinuous antennas are strip ones; that is, gray area in Figure 1, representing one of the antenna arms, is a metal patch over a dielectric substrate, whereas in a slot-sinuous antenna, the gray area in Figure 1 represents a hole etched in on a metallic ground, possibly over a dielectric slab.

To attain unidirectional radiation, strip-sinuous antennas are usually backed with a cavity filled with an absorbing material $[16,21]$. This causes an efficiency drop of at least $50 \%$ and a corresponding lowering in Gain.

Better results would be attained by backing the antenna with a conducting plane acting as a mirror. For the mirror to have its maximum effect the mutual distance between the antenna and the reflecting ground must be one quarter wavelength. If this is trivial in narrow-band applications it is a requirement impossible to meet in wide and ultra wide band applications.

In this particular case, being the antenna working in an active region mode, where just one or two of the bend in each arm of the sinuous strip do actually radiate, it is possible to devise some solution to obtain a reflecting mirror at a quarter wavelength from the active region.

Two solutions are presented here, in the first one several concentric cylindrical shells of dielectric materials are placed beneath the substrate on which the antenna is printed. All cylindrical shells share the same height $h$ and the reflecting ground plane is placed at the bottom. Eight cylindrical shells are considered; their inner and outer radius $r_{p}$ and $r_{p+1}$ ratio is still $\tau$ as for the sinuous antenna. Each shell permittivity and permeability is tuned so that the height $h$ is $\lambda / 4$ at the frequency for which the bend of the sinuous strip immediately above the shell is actively radiating (Figure 2 and Table 1). High losses materials are exploited to attain a better frequency behavior. The corresponding gain diagrams are reported in Figure 3. 


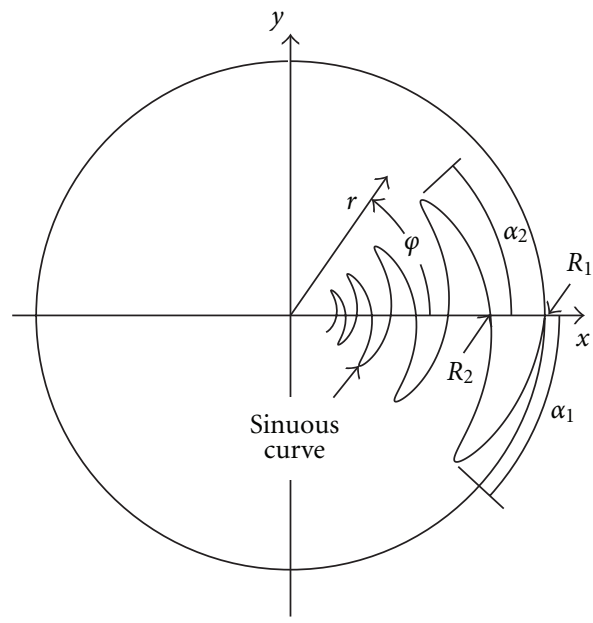

(a)

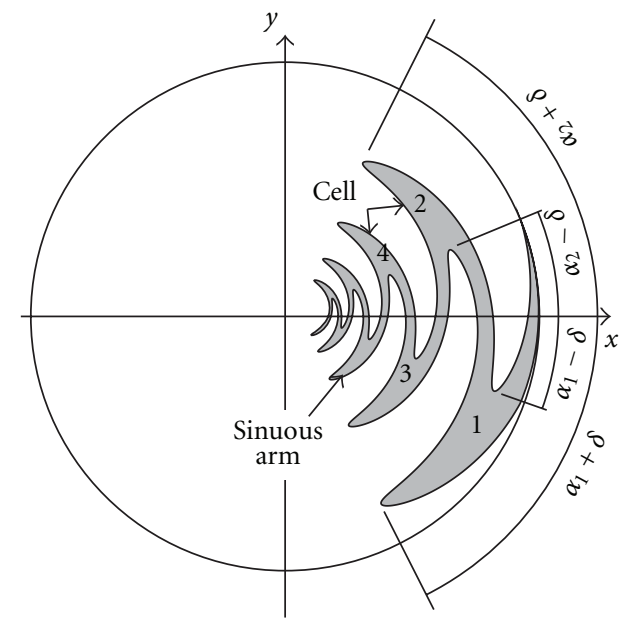

(b)

FIGURE 1: Sinuous curve design and relative parameters.

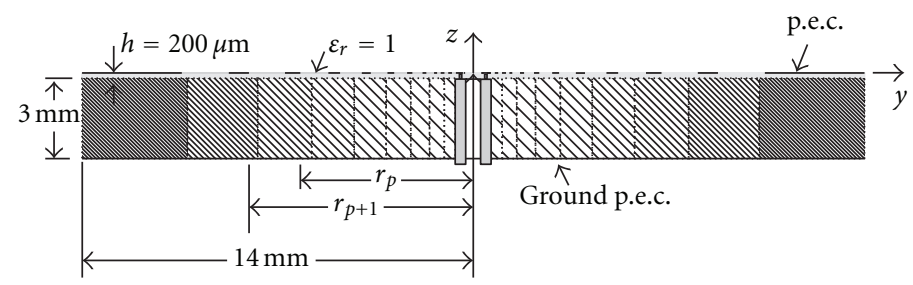

FIGURE 2: Sinuous antenna over a substrate comprising concentric dielectric cylinders of varying permittivity.

TABLE 1: Material parameters.

\begin{tabular}{lcccccccc}
\hline Shell & 0 (inner) & 1 & 2 & 3 & 4 & 5 & 6 & 7 (outer) \\
\hline$\varepsilon_{r}$ & 1 & 1 & 1.3 & 2.3 & 3.8 & 6.5 & 10.9 & 18.36 \\
$\tan \delta$ & 0.5 & 0.5 & 0.5 & 0.5 & 0.5 & 0.5 & 0.5 & 0.5 \\
\hline
\end{tabular}

Figure 3 also reports the patterns for the corresponding-free space-sinuous antenna, whose gain is about $5.5 \mathrm{~dB}$ in circular polarization. This proposed design is generally better than the free space sinuous for what concerns polarization purity, being the cross-polar components lower, but losses introduced in the dielectric lower the gain, especially at lower frequencies, where fields fill a larger volume of dielectric. At best, at high frequencies, the gain is equal to that of the free space sinuous, since all the back lobe power is absorbed by the dielectric.

Finally, in Figure 4 the reflection coefficient of one of the four arms is presented. As it can be seen in Figure 2 the sinuous arms are fed via four via holes in the $200 \mu \mathrm{m}$ substrate, the $3 \mathrm{~mm}$ thick cavity is crossed by four coaxial cables, whose inner conductor is connected to the sinuous arms, while the shields are mutually connected. A suitable modal expansion is hence enforced at the far end of the coaxial cables, beneath the ground plane, and $S_{11}$ computed there.

The second solution presents a conical ground plane, tailored so as to be at one quarter wavelength from the active region for each frequency in the band (Figure 5), and without any losses.

To reduce the cone height a dielectric of high permittivity might be used, but for the sake of easiness of construction and low costs a Rogers RT/duroid $5880\left(\varepsilon_{r}=2.2\right)$ has been employed. Results are reported in Figure 6; curves show a gain comparable with that of the free space sinuous, but much wider $3 \mathrm{~dB}$ beams, thanks to the defocusing properties of the conical reflector. Cross-polar is better than that in the in free space sinuous at lower frequencies and comparable or slightly worse at higher frequencies.

Even more compact structures might be attained by resorting to a ground realized by a perfect magnetic conductor very close to the strip. This can be realized, in narrow band, by a periodic arrangement of metallic patches which realizes the metamaterials properties of a magnetic conductor. If patches' dimensions are varied akin to what is done in the first setup for permittivities then the ground can be tuned to work at the frequency at which the arm of the sinuous above is active. 

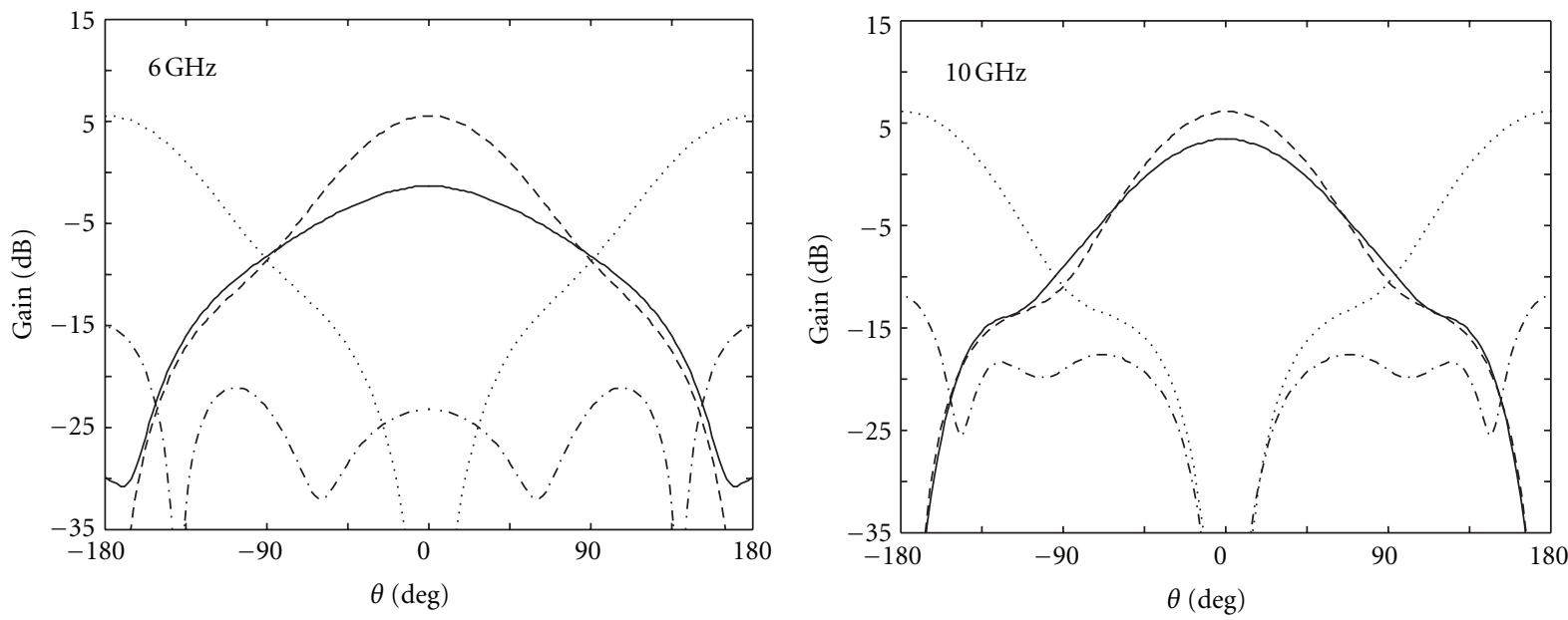

$\begin{array}{lll}\text { _- Proposed design, LHCP } & --- & \text { Reference, } \text { LHCP } \\ \text { _. } & \text { Proposed design, RHCP } \quad \ldots . . . & \text { Reference, RHCP }\end{array}$

(a)

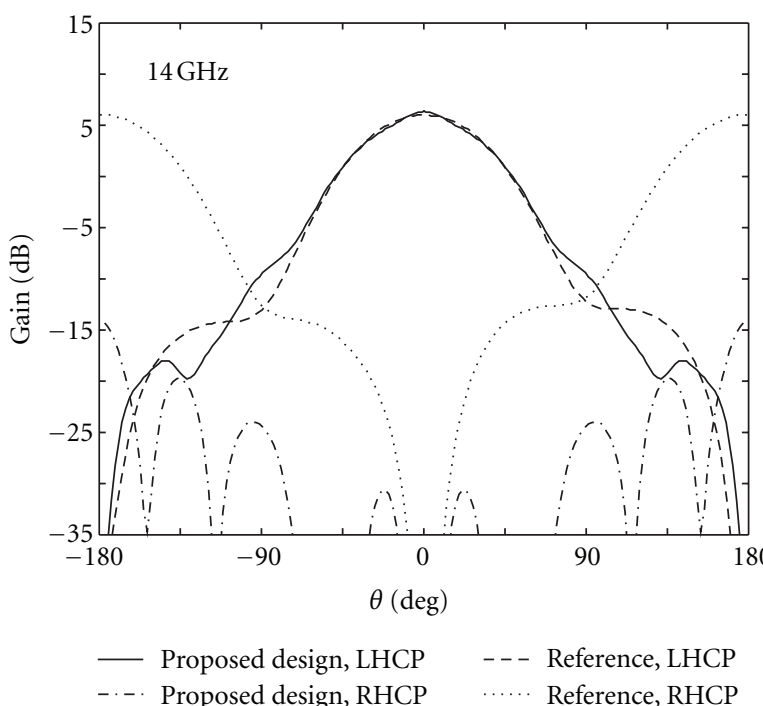

(c)

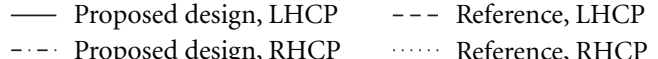

(b)
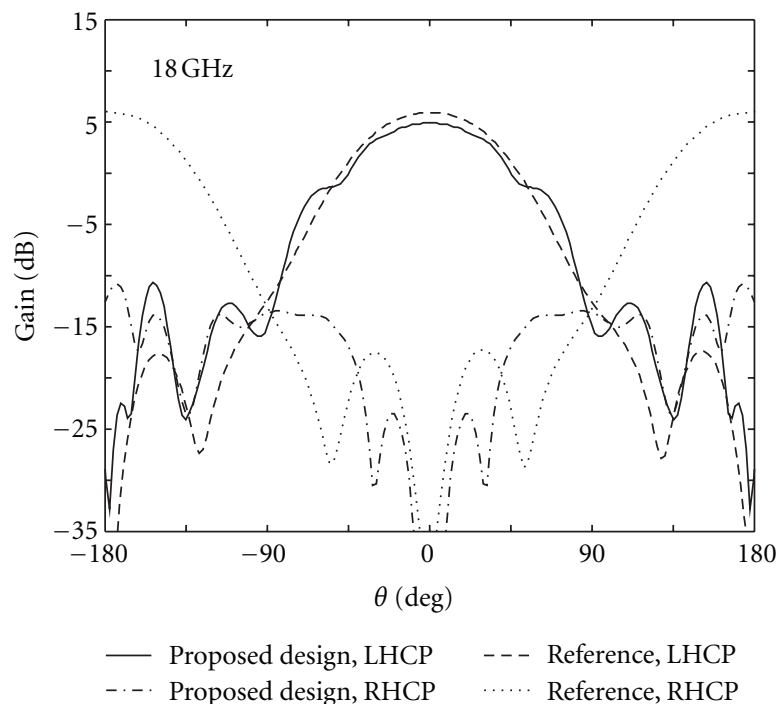

(d)

Figure 3: Simulated Gain diagrams of the structure backed with lossy concentric dielectric cylinders, at $\phi=0$ compared to the free space sinuous.

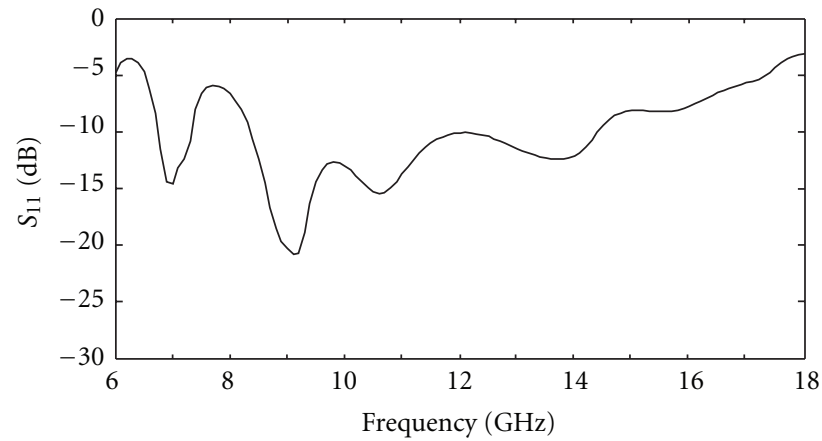

FIGURE 4: $S_{11}$ at the feed port for any of the four sinuous arms. 


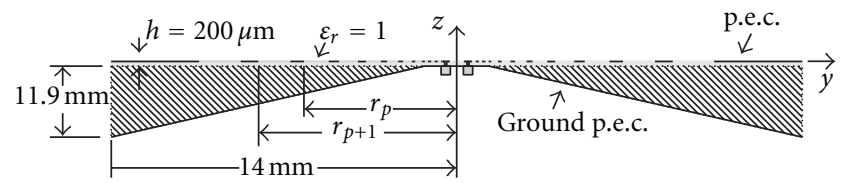

FIGURE 5: Sinuous antenna over a conical ground plane comprising a dielectric loading.

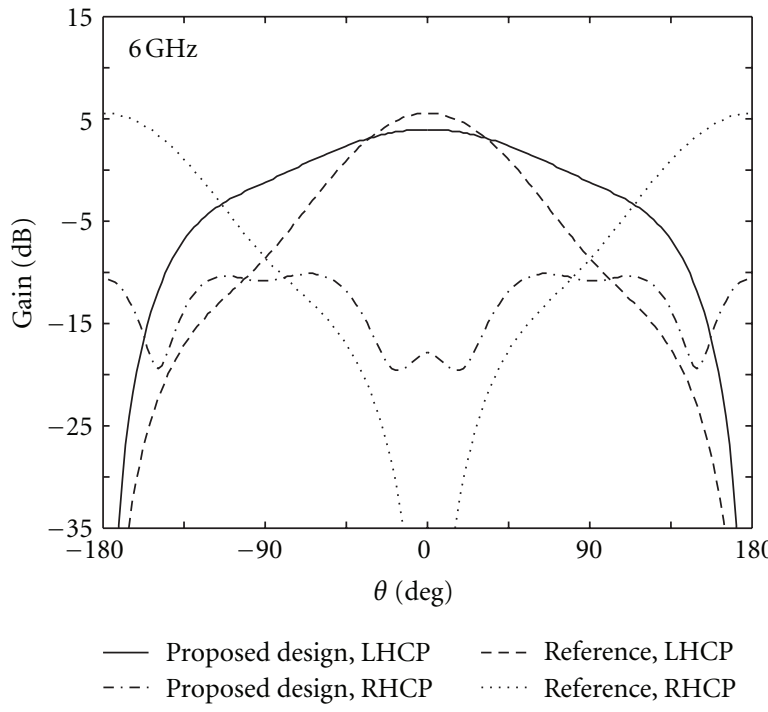

(a)

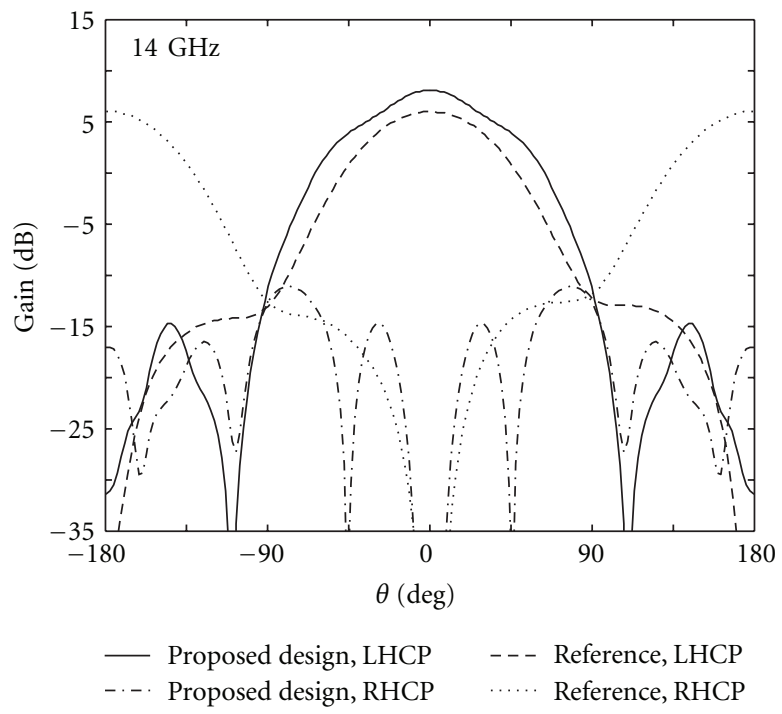

(c)

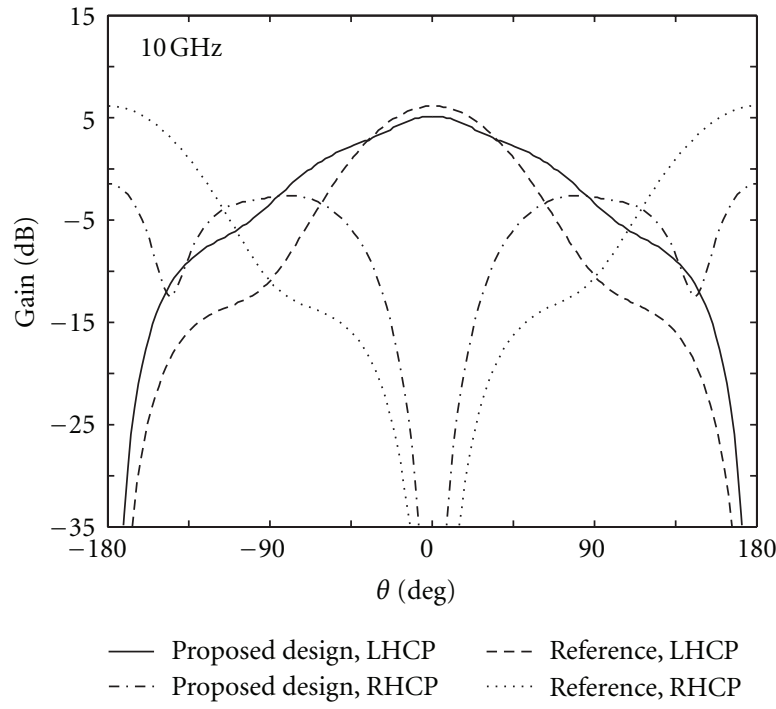

(b)

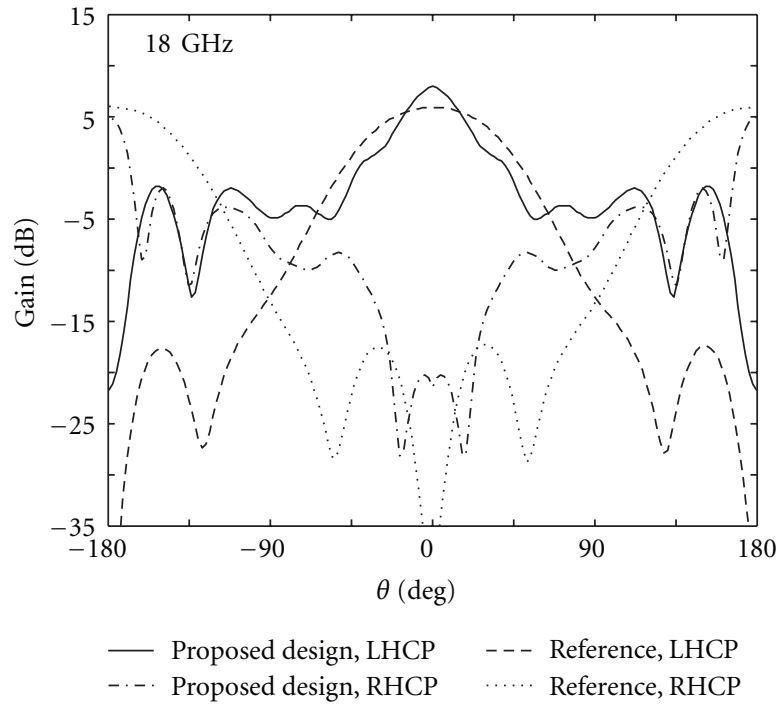

(d)

FIGURE 6: Simulated far-field patterns of the structure backed by a conical ground plane $\phi=0$ compared to the free space sinuous antenna pattern.

\section{Slim Slot Configuration}

To overcome the aforementioned issues, nor a large lossy cavity nor an artificial metamaterials implementing a perfect magnetic conductor is necessary. Whereas radiating sources in a standard sinuous are electric currents, which does not radiate if placed in the vicinity of a ground plane, radiating sources in a slot-sinuous are the equivalent magnetic currents on the slots, hence a ground plane can be brought closer to the slots.

The presence of the ground plane close to the slots ideally will increase the current amplitude by a factor of two. Consequently the radiated electric field and maximum gain are expected to grow at most of $6 \mathrm{~dB}$ with respect 


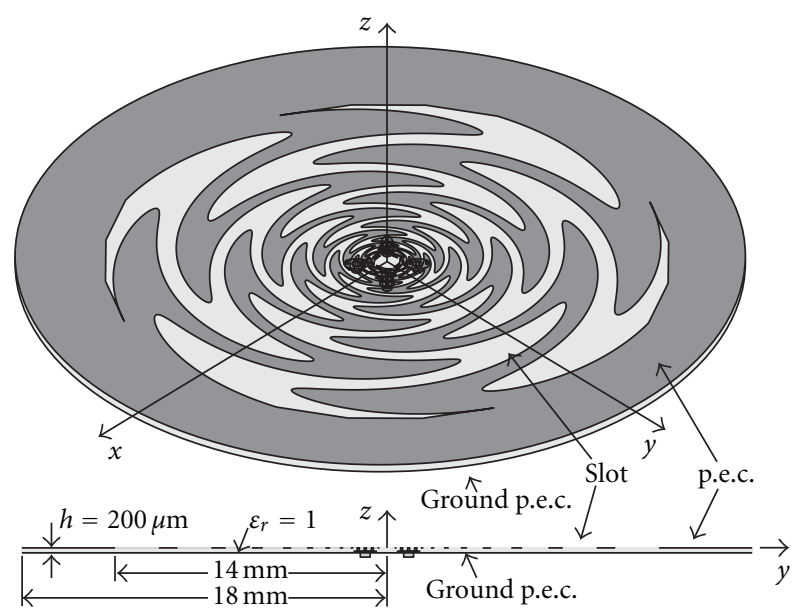

FIgURE 7: 3D model of the analysed slot-sinuous structure and dimensions.

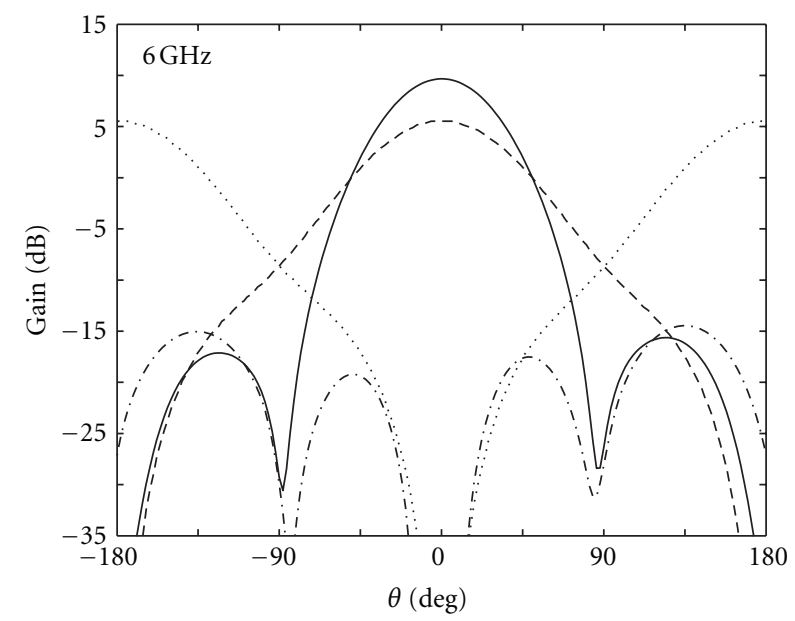

— Proposed design, LHCP

-. - Proposed design, RHCP

(a)

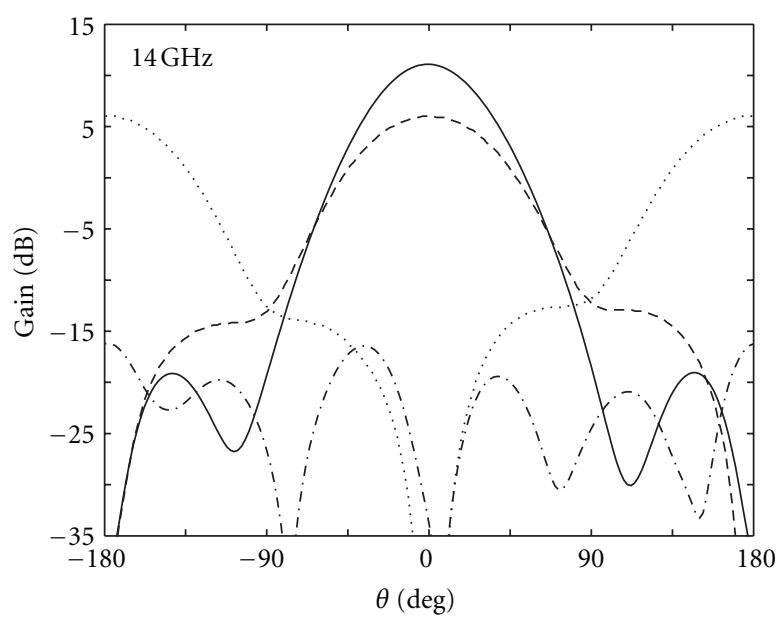

$\begin{array}{llll}\text { —- Proposed design, LHCP } & --- & \text { Reference, } \text { LHCP } \\ \text {-.- Proposed design, RHCP } & \ldots . . & \text { Reference, RHCP }\end{array}$

(c)

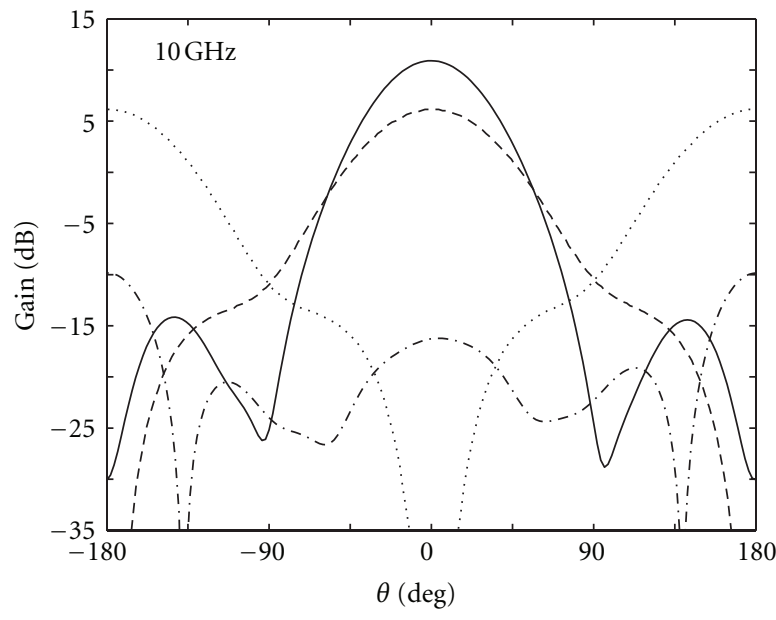

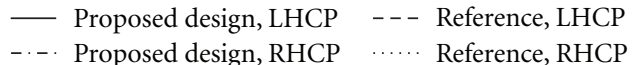

(b)

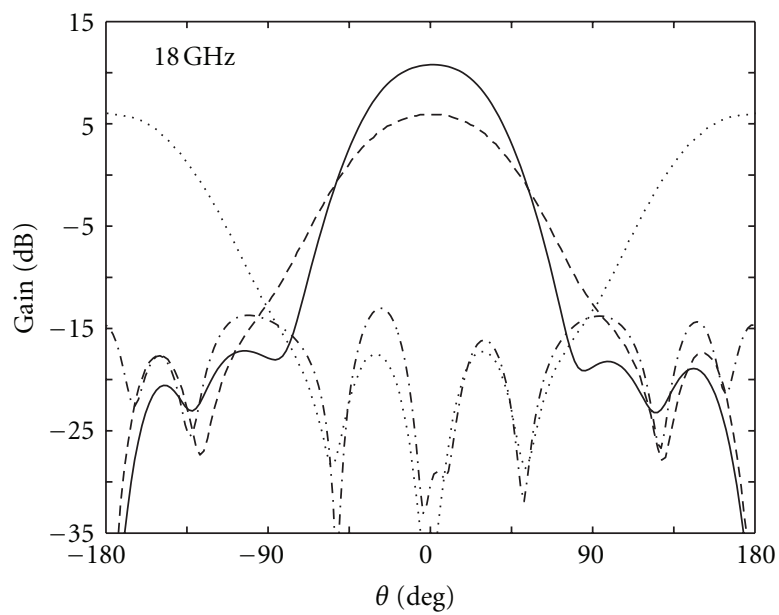

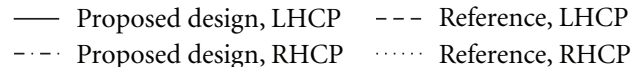

(d)

FIGURE 8: Simulated far-field patterns of the proposed slot structure at $\phi=0$, compared to the free space sinuous antenna pattern. 


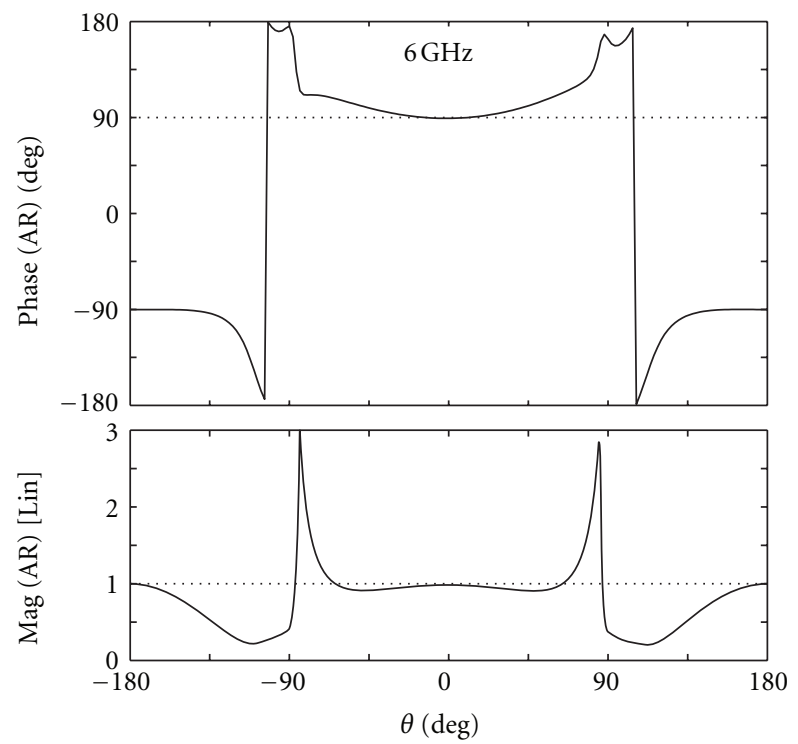

(a)

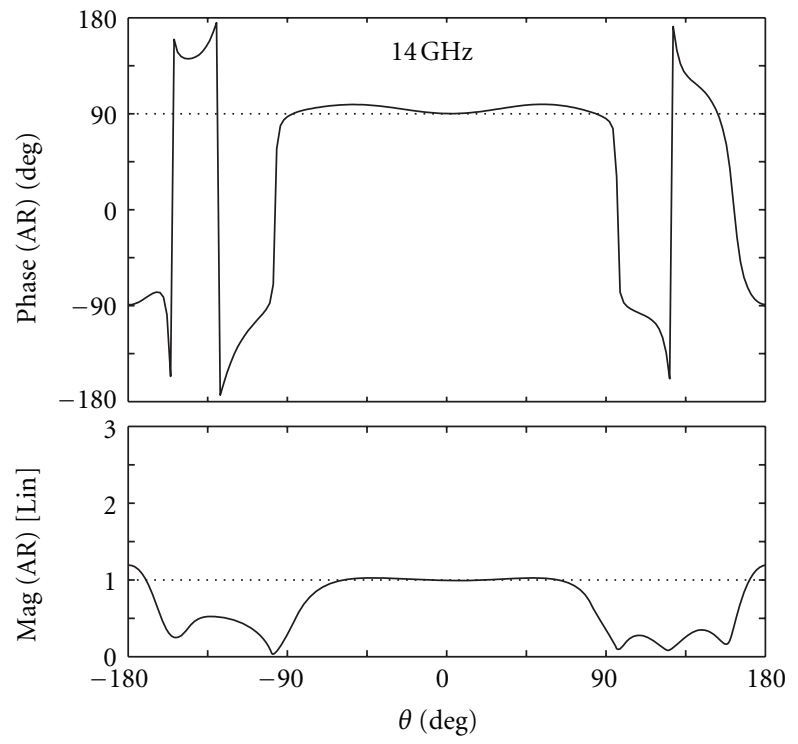

(c)

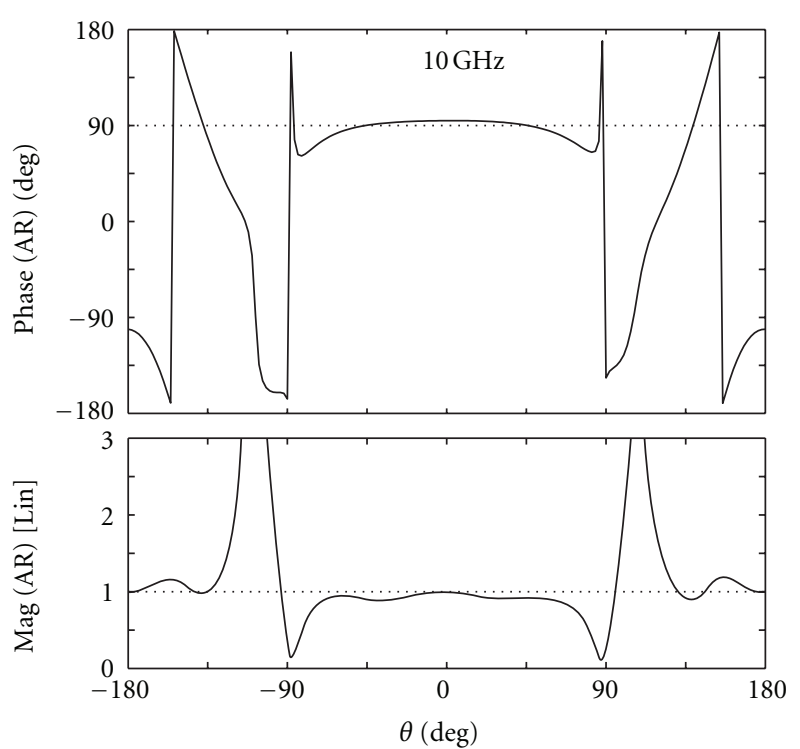

(b)

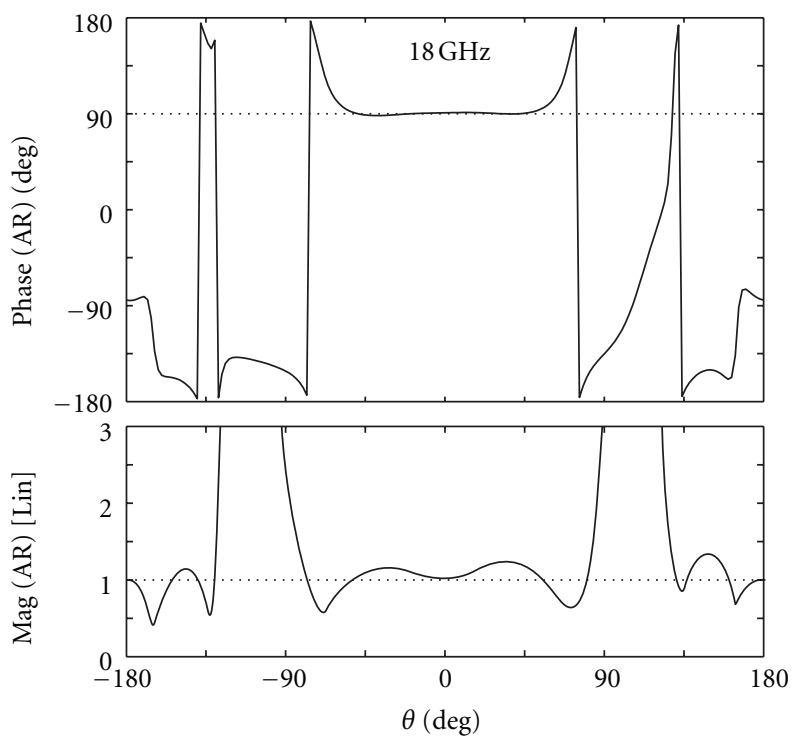

(d)

FIgURE 9: Axial ratio (AR) and phase between the two orthogonal components for the proposed slot structure.

to the standard sinuous antenna in free space, whose gain is about $5.5 \mathrm{~dB}$ in circular polarization. The slot-sinuous presented here, thanks to the ground plane, should hence present a maximum peak gain of about $11.5 \mathrm{~dB}$.

In general, placing the ground plane at less than $\lambda / 4$ at the highest frequency leads to a constructive sum of direct and reflected radiation in the upper space.

In this paper the ground plane to slot plane distance $h$ is varied from $200 \mu \mathrm{m}$ to $1 \mathrm{~mm}$ to show the effect of the ground on the antenna parameters. Such distances are about 83 to 16 times smaller than the smallest wavelength in free space $(\approx 16.7 \mathrm{~mm} @ 18 \mathrm{GHz})$. A dielectric of $\varepsilon_{r}=1$ is chosen. Other relevant geometrical parameters are inserted in Figure 7.
In Figure 8 the gain of the proposed structure for the ground to slot distance $h=200 \mu \mathrm{m}$ is plotted for four representative frequencies in the range $6-18 \mathrm{GHz}$, both for LHCP (copolar) and RHCP (cross-polar). Cuts are presented on the $\phi=0$ plane for the sake of brevity, as antenna symmetry leads to substantially equal results on the orthogonal $(\phi=$ $90^{\circ}$ ) and any other plane. Similar results are obtained for $h=$ $600 \mu \mathrm{m}$ and $h=1 \mathrm{~mm}$, again not presented here for brevity. To better compare the proposed structure to the standard one, Figure 8 presents also the gain for the free space, not ground backed, sinuous slot structure. This better gain and extreme thinness is in a trade-off with the antenna reflection coefficient, which is slightly worse than in the previous cases. 


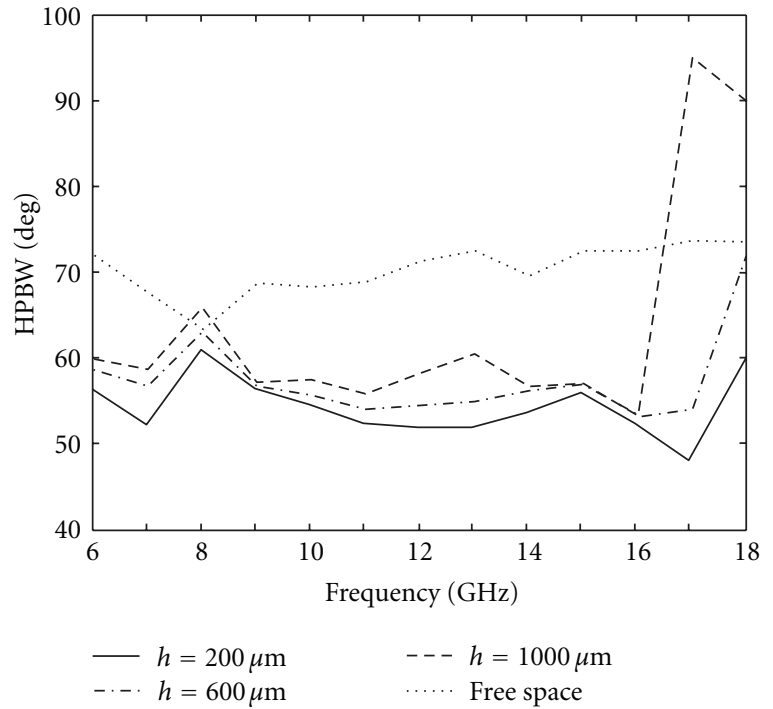

(a)

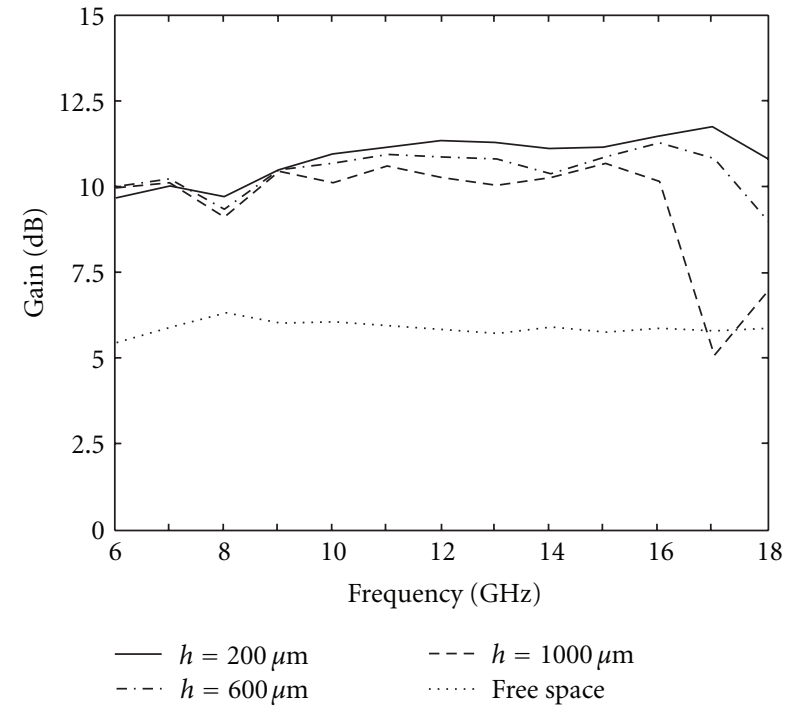

(b)

FIGURE 10: HPBW and maximum gain in the frequency band of the proposed slot structure for three different distances $h$, compared to the free space standard one.

To evaluate the circular polarization purity, the axial ratio (AR) and the phase between the two orthogonal electric field components $E_{\theta}$ and $E_{\phi}$ are shown, at the same four frequencies, in Figure 9. The phase of $-90^{\circ}$ observed for back radiation indicates a reversal of polarization for back radiation. Anyway, the front to back ratio is less than $-20 \mathrm{~dB}$ for this design. Both the axial ratio and the phase clearly indicate the transition from linear polarization $(\mathrm{AR}=0$, or $\mathrm{AR}=\infty$ and phase $=0$ or $\pm 180^{\circ}$ ) to circular polarization (AR $=1$ and phase $= \pm 90^{\circ}$ ).

The presented structure shows a mean gain around $11 \mathrm{~dB}$ and a HPBW around 50-60 deg. Modifying the ground plane distance from the slot aperture, a limited control on the HPBW can be obtained as Figure 10 shows. The high variation visible around $17-18 \mathrm{GHz}$ for the highest $h$ value is due to some direct radiation from the feeding pins, which are designed similar to those in $[18,19]$.

Finally Figure 11 shows a comparison between the peak realized gain of the three structures. The best being the slot configuration, followed by the strip sinuous with conical ground, where no losses are present but the conical reflector causes a widening of the beam. The worse being of course the strip sinuous backed by the lossy dielectrics.

\section{Conclusion}

In this paper three broadband low-profile cavity-backed sinuous antennas have been presented. The two cavity backed configurations present an overall diameter of $28 \mathrm{~mm}$ and a thickness of $3 \mathrm{~mm}$ or $12 \mathrm{~mm}$; the slot configuration has an overall diameter of $36 \mathrm{~mm}$ and a smaller thickness, all configurations working from 6 to $18 \mathrm{GHz}$. The attained nominal gain of the best configuration, the slot one, is greater than $10 \mathrm{~dB}$ and excellent circular polarization properties are

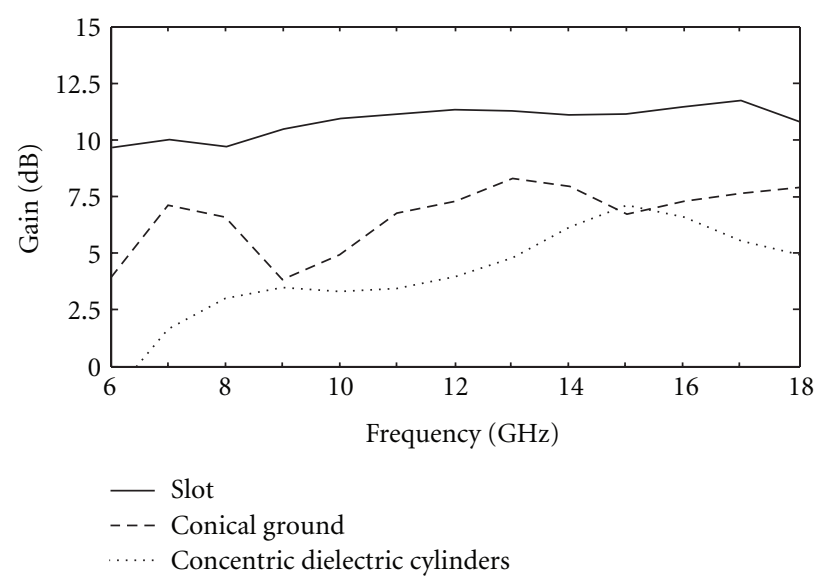

Figure 11: Maximum gain as a function of frequency for the slotsinuous with $200 \mu \mathrm{m}$ substrate compared with the maximum gains attained by the strip sinuous with conical ground, and the strip sinuous with concentric cylinders of varying permittivities in the backing cavity.

shown. Full wave analysis was carried out exploiting FEM. Structures with greater radius can allow for lower operational frequencies and hence larger bandwidths. Furthermore, a limited control on some antenna parameters (HPBW) has been shown to be possible by varying the ground distance.

\section{References}

[1] Z. N. Chen and M. Y. W. Chia, Broadband Planar AntennasDesign and Applications, John Wiley \& Sons, Hoboken, NJ, USA, 2006.

[2] M. N. Afsar, Y. Wang, and R. Cheung, "Analysis and measurement of a broadband spiral antenna," IEEE Antennas and Propagation Magazine, vol. 46, no. 1, pp. 59-64, 2004. 
[3] X. M. Wang, L. Zhang, J. P. Xiong, and Y. C. Jiao, "Printed microstrip-line-fed elliptical slot antenna with very wide bandwidth," Journal of Electromagnetic Waves and Applications, vol. 22, no. 14-15, pp. 1865-1871, 2008.

[4] T. Hong, S. X. Gong, W. Jiang, Y. X. Xu, and X. Wang, "A novel ultra-wide band antenna with reduced radar cross section," Progress in Electromagnetics Research, vol. 96, pp. 299-308, 2009.

[5] D. Chen and C. H. Cheng, "A novel compact ultra-wideband (UWB) wide slot antenna with via holes," Progress in Electromagnetics Research, vol. 94, pp. 343-349, 2009.

[6] A. A. Lotfi Neyestanak, "Ultra wideband rose leaf microstrip patch antenna," Progress in Electromagnetics Research, vol. 86, pp. 155-168, 2008.

[7] A. Mehdipour, K. Mohammadpour-Aghdam, and R. FarajiDana, "Complete dispersion analysis of vivaldi antenna for ultra wideband applications," Progress in Electromagnetics Research, vol. 77, pp. 85-96, 2007.

[8] A. A. Eldek, A. Z. Elsherbeni, and C. E. Smith, "Rectangular slot antenna with patch stub for ultra wideband applications and phased array systems," Progress In Electromagnetics Research, vol. 53, pp. 227-237, 2005.

[9] G. Cappelletti, D. Caratelli, R. Cicchetti, and M. Simeoni, "A low-profile printed drop-shaped dipole antenna for wideband wireless applications," IEEE Transactions on Antennas and Propagation, vol. 59, no. 10, pp. 3526-3535, 2011.

[10] J. Y. Sze, T. H. Hu, and T. J. Chen, "Compact dual-band annular-ring slot antenna with meandered grounded strip," Progress in Electromagnetics Research, vol. 95, pp. 299-308, 2009.

[11] T. A. Milligan, "Frequency-independent antennas," in Modern Antenna Design, pp. 521-571, IEEE Press, John Wiley \& Sons, Hoboken, NJ, USA, 2nd edition, 2005.

[12] Q. Liu, C. L. Ruan, L. Peng, and W. X. Wu, "A novel compact archimedean spiral antenna with gap-loading," Progress in Electromagnetics Research Letters, vol. 3, pp. 169-177, 2008.

[13] R. H. DuHamel, "Dual polarized sinuous antenna," US Patent $4658262,1987$.

[14] J. P. Scherer, "The dual polarized sinuous antenna," Journal of Electronic Defense, vol. 13, no. 8, pp. 73-75, 1990.

[15] T. Chu and H. G. Oltman Jr., "The sinuous antenna," Microwave Systems News and Communications Technology, vol. 18, pp. 40-48, 1988.

[16] Y. Yan and Q. Cao, "Microwave absorbing properties of sinuous antenna filled with absorbing material," in Proceedings of the IEEE International Conference on Ultra-Wideband (ICUWB'10), vol. 1, pp. 1-4, September 2010.

[17] S. Palreddy, A. I. Zaghloul, and R. Cheung, "An optimized lossy back cavity loaded four arm sinuous antenna," in Proceedings of the IEEE Antennas and Propagation Society International Symposium (APSURSI), pp. 1-4, Toronto, Canada, July 2010.

[18] M. C. Buck, J. Burford, and D. S. Filipović, "Multiband two arm slot sinuous antenna," in Proceedings of the IEEE Antennas and Propagation Society Symposium, pp. 165-168, June 2004.

[19] M. C. Buck and D. S. Filipović, "Split-beam mode four-arm slot sinuous antenna," IEEE Antennas and Wireless Propagation Letters, vol. 3, no. 1, pp. 83-86, 2004.

[20] E. Agastra, "A high gain slot Sinuous antenna," Atti della Fondazione Giorgio Ronchi, vol. 66, no. 5, pp. 635-639, 2011.

[21] L-3 Communications, Randtron Antenna Systems, http:// www.l-3com.com/randtron/dcpantenna.htm. 

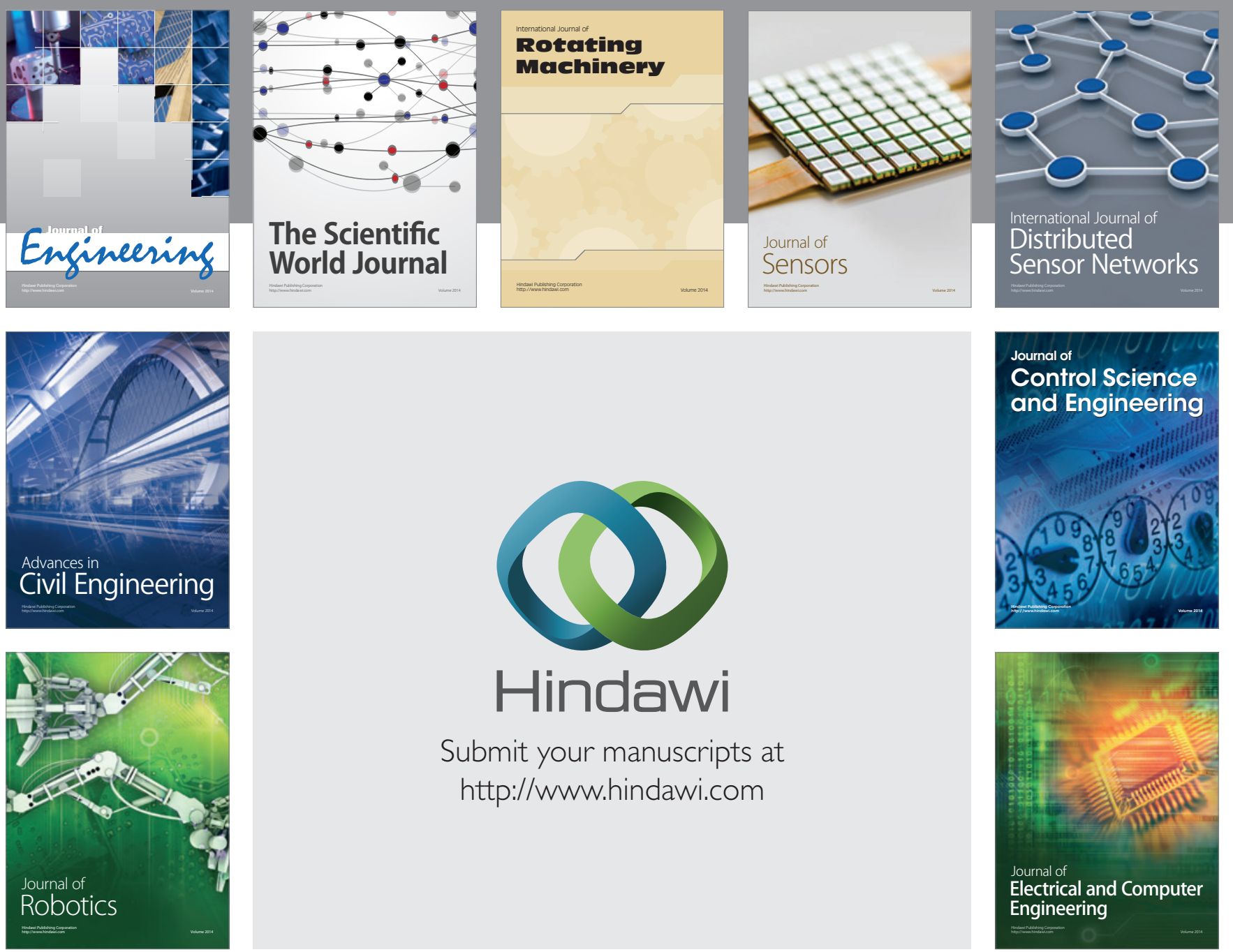

Submit your manuscripts at

http://www.hindawi.com
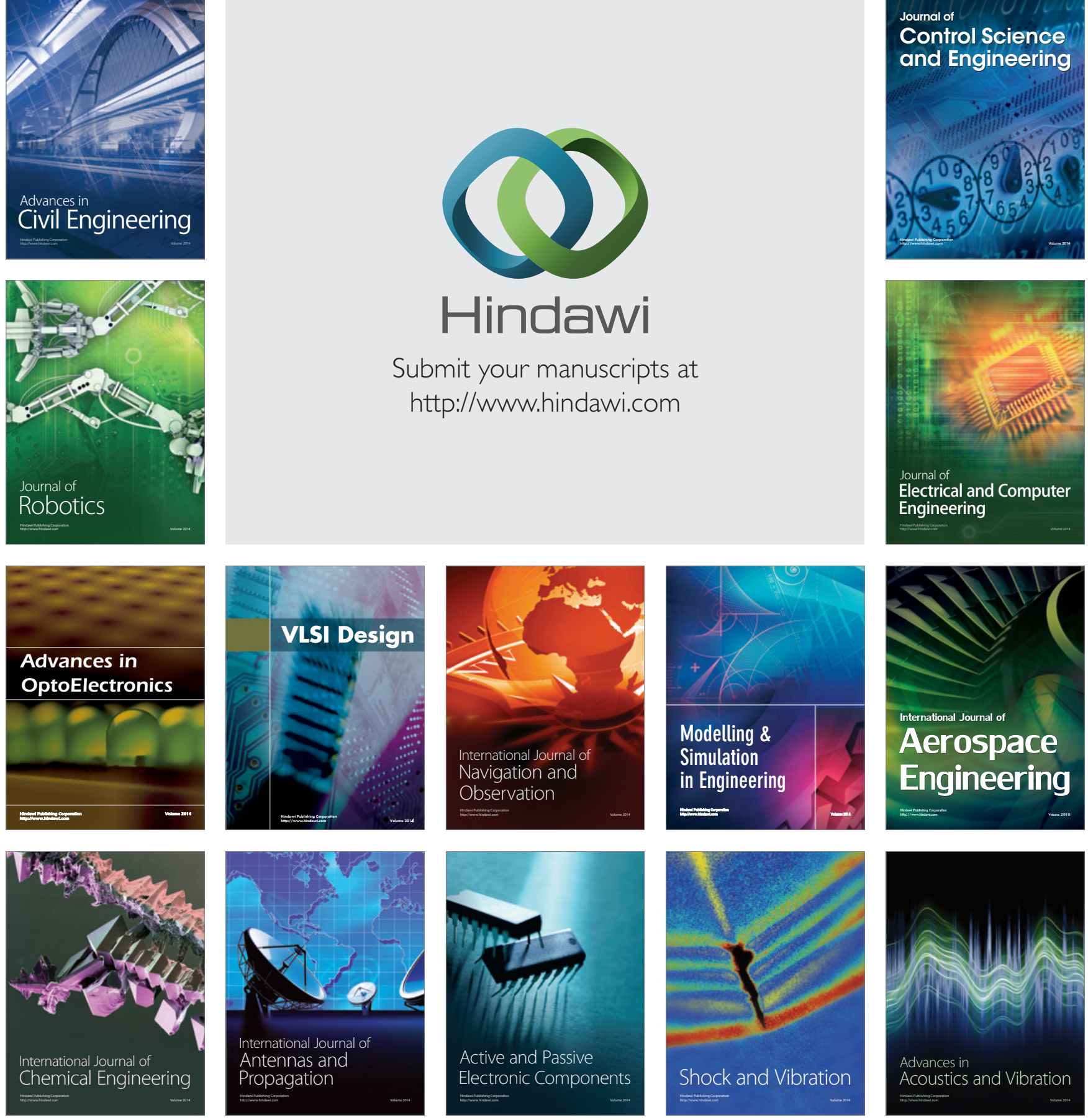